\title{
An Unusual Presentation of Recurrent Hurthle Cell Carcinoma of Thyroid
}

\author{
Saeed Akhter ${ }^{1}$, Hira Andleeb ${ }^{1 *}$ and Sobia Tabassum² \\ 'Department of ENT and Head and Neck Surgery, Liaquat National Hospital and Medical College, Karachi, Pakistan \\ ${ }^{2}$ Department of Oncology, Liaquat National Hospital and Medical College, Karachi, Pakistan
}

\begin{abstract}
This is the case of 55 years old male who was known of hurthle cell carcinoma (HCC) and underwent total thyroidectomy and right neck dissection in July 2015, followed by radioactive iodine (RAI). He was in close follow-up after the treatment when he presented with right sided neck swelling for 6 weeks followed by hoarseness for 4 weeks and fiber optic laryngoscopy revealed right vocal cord palsy. CT scan was done that showed a mass involving right supraglottic and glottis region, he then underwent right vertical partial laryngectomy. Tumor removed completely (grossly and histopathologically). The case was discussed in tumor board meeting and he was advised RAI scan to look for residual disease, by close follow-up. This case report emphasizes on the unusual clinical presentation and recurrence of HCC of thyroid.
\end{abstract}

Keywords: Hurthle cell carcinoma, thyroid, oncocytic carcinoma.

\section{INTRODUCTION}

Hurthle cell carcinoma (HCC) of the thyroid, also called Oncocytic carcinoma, is a rare form of differentiated thyroid cancer. Preoperative clinical, cytological, and genetic studies have not shown to reliably discriminate between benign and malignant variants of hurthle cell neoplasms $(\mathrm{HCN})$. Therefore, histopathological analysis remains the gold standard for diagnosis; although, it may be apparent preoperatively if there is evidence of metastasis [1]. The overall recurrence rate $12.1 \%$, with average time for relapse of 90.74 months and average time without any signs of the disease of 222.4 months. Overall 5-year, 10-year and 20-year survival rates are $89.4 \%, 77.2 \%$ and $61.9 \%$ respectively [2].

\section{HISTORY}

This is a case of 55 years old male, who underwent total thyroidectomy along with right modified radical neck dissection and central compartment clearance done at LNH in July 2015. Neck dissection was planned on the basis of clinically palpable multiple lymph nodes at right level 2, 3 and 4. Final H/P showed hurthle cell carcinoma, involving the right lobe and thymus measuring $8.5 \times 7 \times 5 \mathrm{~cm}$, sparing left lobe with no lymphovascular invasion or extra capsular spread. There was no metastasis to lymph nodes.

The case was then discussed in tumor board and radioactive iodine ablation was advised. He underwent RAl ablation and was on close follow up as an outpatient till July 2019, when he presented with right sided neck swelling measuring $4 \times 5 \mathrm{~cm}$ which was non tender, firm,

*Corresponding Author: Hira Andleeb, Department of ENT and Head and Neck Surgery, Liaquat National Hospital and Medical College, Karachi, Pakistan;Email:hira_andleeb@yahoo.com

Received: January 05, 2020; Revised: June 24, 2020; Accepted: July 12, 2020

DOI: https://doi.org/10.37184/lntbj.2708-7808.2.8 fixed mass overlying right thyroid cartilage. U/S neck showed a heterogeneous area measuring $2.4 \times 1.9 \mathrm{~cm}$ in right side of neck with increased vascularity, suspicious of a neoplastic lesion. His RAI scan showed no residual functioning thyroid tissue. Fine needle aspiration cytology (FNAC) was done which showed suspicion of hurthle cell neoplasm (Thy III Bethesda 4).

He then developed hoarseness of voice after 15 days of presentation of the neck swelling, which was persistent. Fiber-optic laryngoscopy was done as an outpatient that revealed right vocal cord palsy but no obvious mucosal lesion.

\section{RADIOLOGICAL FINDINGS}

CT scan neck and chest was done. It showed absence of previous large mass in right lobe of thyroid, sternocleidomastoid, internal jugular vein and submandibular gland, due to previous neck dissection. Small sized left lobe of thyroid measured $1.7 \times 1 \mathrm{~cm}$.

Scan showed a heterogeneously enhancing mass in right glottis and supraglottic region eroding through the thyroid cartilage (Figs. 1\&2). It is causing mild narrowing of the air column, abutting the cricoid and arytenoid cartilages with possible focal erosion. Superiorly this mass is involving the right aryepiglottic fold causing mild obliteration of the pyriform sinus. Laterally it is abutting right common carotid artery with blurring of intervening fat planes. Posteriorly mass was abutting the posterior pharyngeal wall, and anteriorly involving the strap muscles, and measures $3.7 \times 3.5 \times 2.8 \mathrm{~cm}$. Significant surrounding fat stranding is present.

\section{PLAN OF SURGERY}

This case was discussed in tumor board meeting and after discussing the radiological, histopathological and 


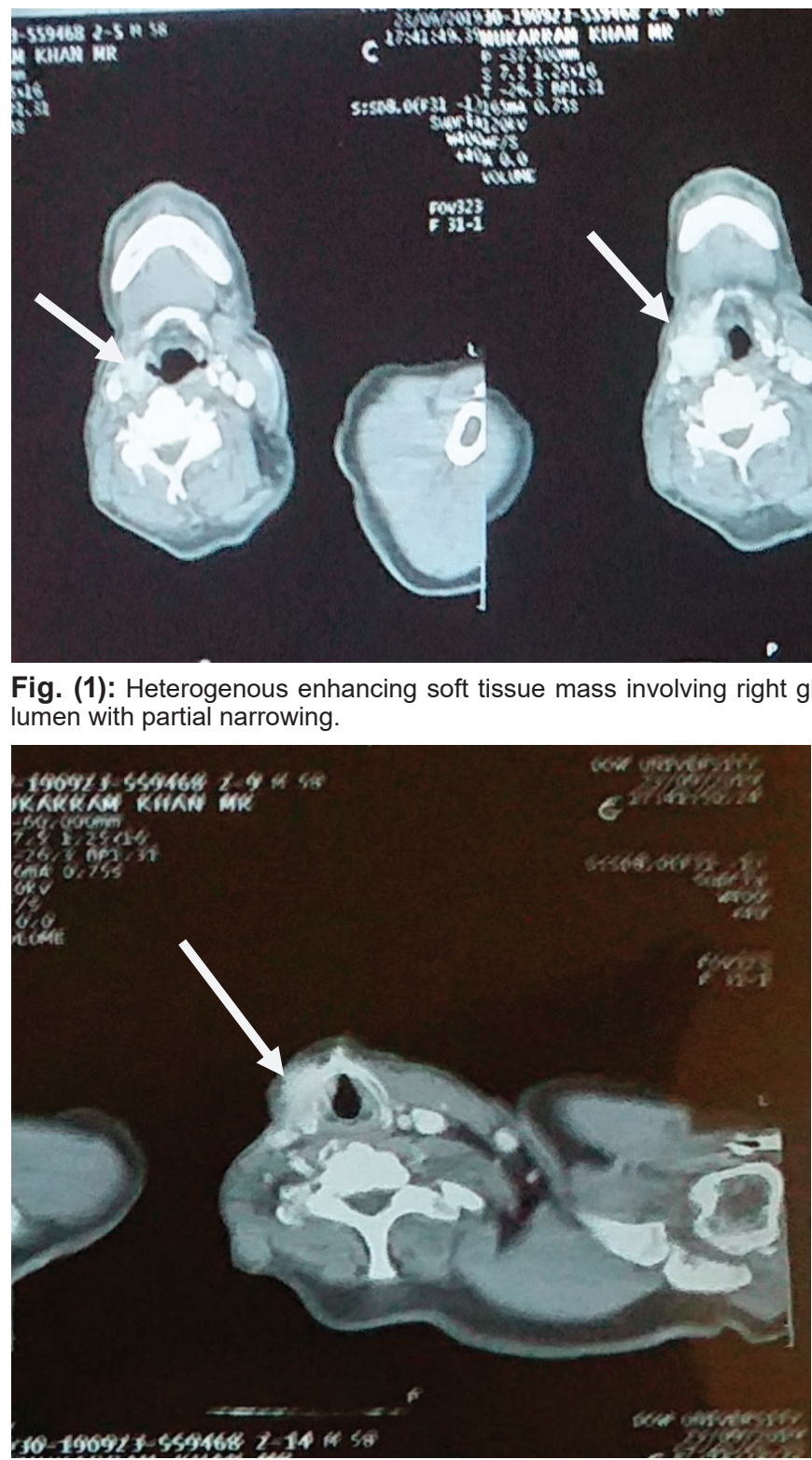

Fig. (2): Inferiorly causing erosion of cricoid cartilage and anteriorly involving the subcutaneous fat planes.

clinical aspects of the tumor, we decided to go for the surgical excision of the tumor as it was possible to remove the tumor. A right partial laryngectomy was planned but as the tumor was also involving the thyroid cartilage and overlying strap muscles there was a need to provide tissue coverage for the right hemilarynx. A pectoralis major flap was to be used for this purpose.

The procedure was done successfully.

\section{INTRAOPERATIVE FINDINGS}

Tumor was involving right thyroid lamina and right paraglottic region (causing bulging of right false and true cord with obliteration of ventricle).

Right sided sternocleidomastoid muscle and strap muscles were absent.
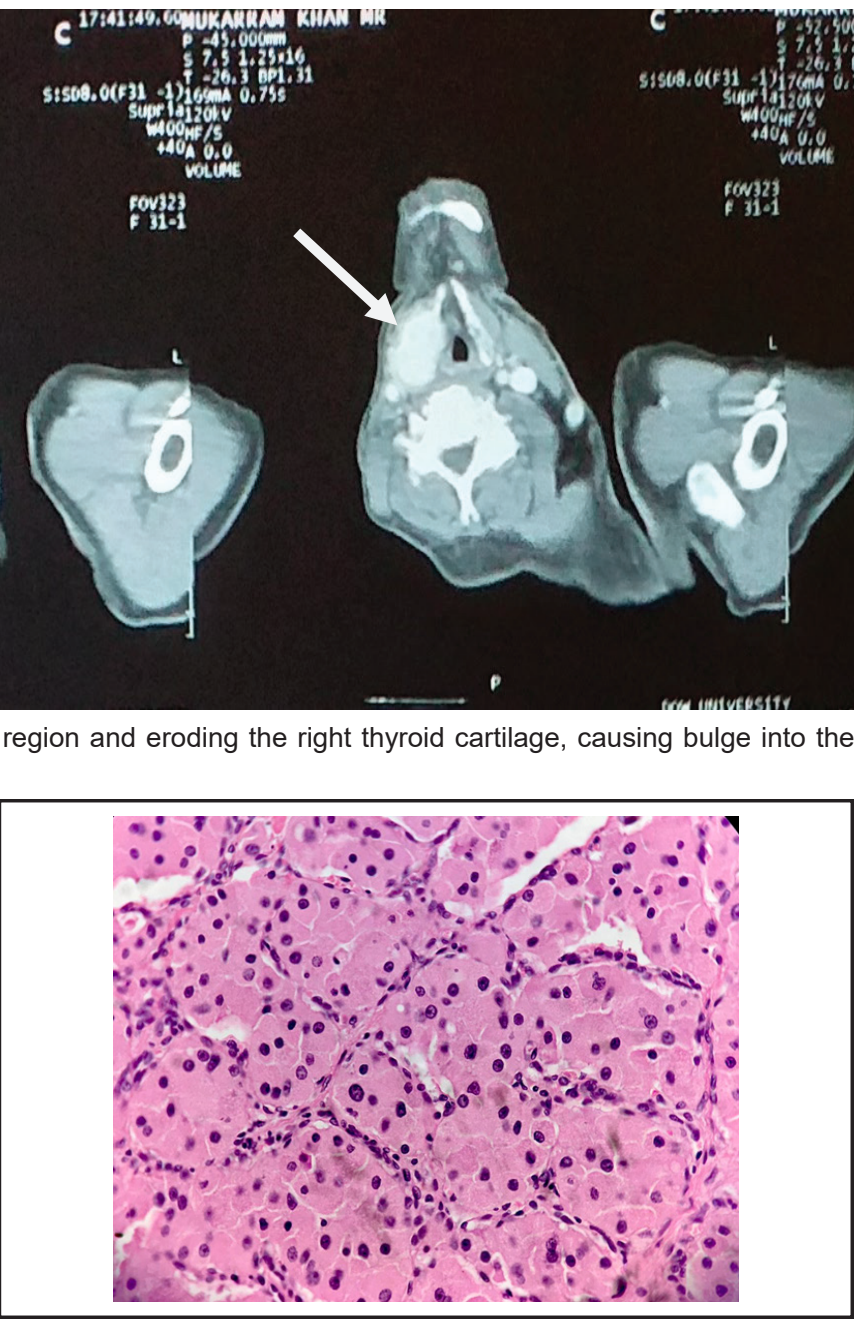

Fig. (3): Hurthle cell carcinoma 40x.

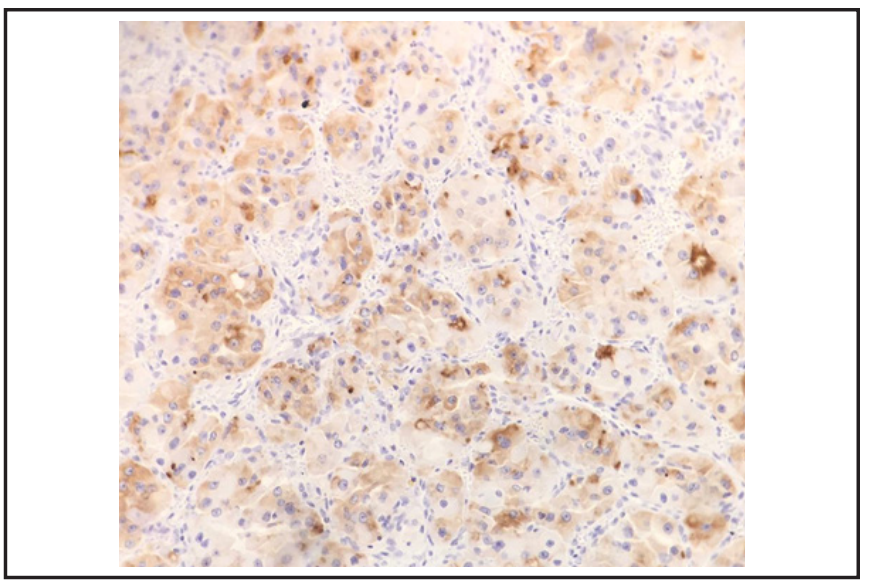

Fig. (4): Thyroglobulin positive.

\section{HISTOPATHOLOGY}

Final histopathology showed residual/ recurrent hurtle cell carcinoma measuring $4 \times 3.4 \times 1.5 \mathrm{~cm}$. Tumor is extensively infiltrating skeletal muscle and thyroid cartilage.

Hürthle cells are thyroid follicular cells with oncocytic appearance characterized by large hyperchromatic nuclei with prominent nucleoli and abundant granular 


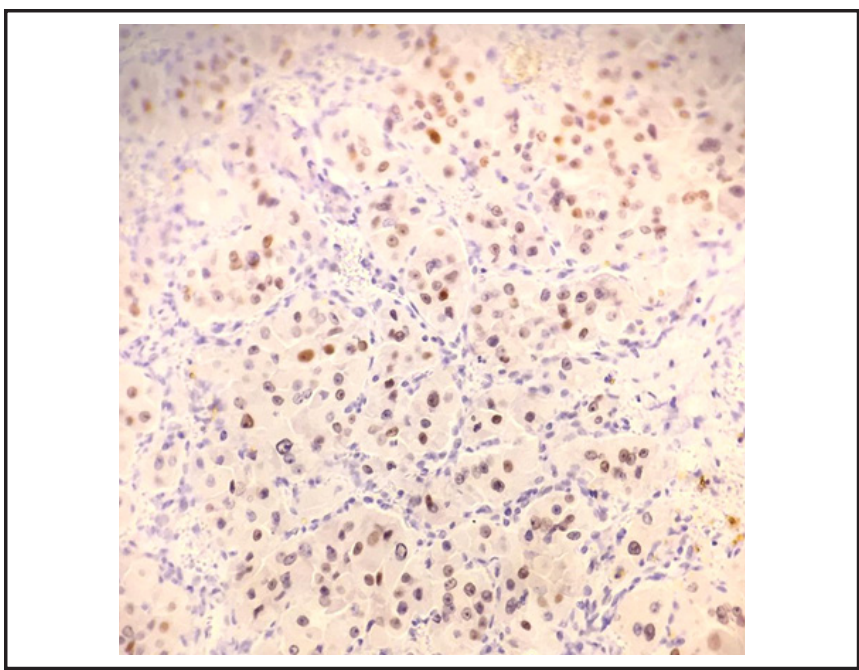

Fig. (5): TTF1 positive.

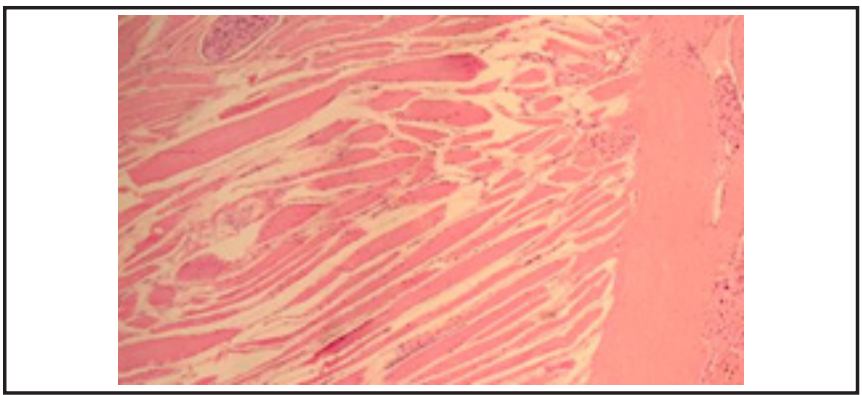

Fig. (6): Tumor infiltrating the thyroid cartilage.

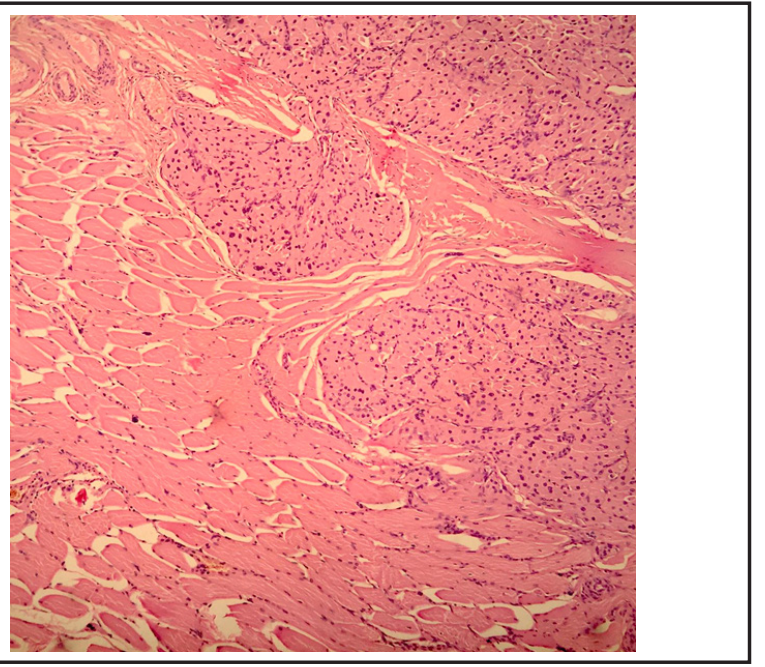

Fig. (7): Tumor infiltrating the skeletal muscles.

eosinophilic cytoplasm stained blue, purple (Fig. 3) and they are thyroglobulin positive (Fig. 4). TTF-1 is a tissue-specific transcription factor expressed mainly in the epithelial cells of the lungs and thyroid (Fig. 5).

\section{Surgical Margins were as follows:}

- Medial mucosal margin: tumor free (0.4 cm away).

- Superior mucosal margin: tumor free $(0.5 \mathrm{~cm}$ away).

- Inferior mucosal margin: tumor free $(0.4 \mathrm{~cm}$ away).

- Anterior soft tissue margin: $0.2 \mathrm{~cm}$ away.

- Lateral soft tissue margin: $0.2 \mathrm{~cm}$ away.
- Posterior soft tissue: tumor free $(0.5 \mathrm{~cm}$ away).

- Tumor infiltrating the thyroid cartilage (Fig. 6).

- Tumor infiltrating the skeletal muscles (Fig. 7).

\section{TUMOR BOARD DISCUSSION}

Case was again discussed in tumor board meeting after surgery and RAI scan was advised to look for residual disease. In case of no residual thyroid tissue, close follow-up was advised as grossly and microscopically tumor was removed completely.

\section{FOLLOW-UP}

Patient came on regular follow up during the postop period and showed good recovery in terms of breathing and swallowing. He was decannulated 6 weeks after the surgery and also nasogastric tube was removed after 8 weeks, his speech is clear and understandable.

RAl scan was done after 6 weeks and it showed no residual disease.

\section{SURGEON'S PERSPECTIVE}

Keeping the radiological, histopathological and gross features in mind, the tumor is stage 4 a (involving the extra thyroidal structures), also his age 55 years, and RAI ablation was already given after the previous surgery when the disease was stage 3 (tumor was more than $4 \mathrm{~cm}$ in size and patient was less than 55 years) [3]. Currently the tumor showed no active uptake on RAI, which is the feature of HCC and in this case giving RAI ablation therapy will not be beneficial.

Since the tumor seems resectable and it is confined to the right side of larynx that is to right thyroid lamina, cricoid cartilage (erosion) but not crossing the midline. By doing a vertical partial laryngectomy postoperative functional status of patient can be good in terms of voice preservation and avoiding a tracheostomy. In cases of squamous cell carcinoma (SCC) of larynx requiring vertical partial laryngectomy the strap muscles are used to cover the wound but since they were involved by the tumor, this patient needed a reconstruction with some other tissue which in this case was pectoralis major muscle flap.

\section{ONCOLOGIST'S PERSPECTIVE}

Hurthle cell carcinoma is usually assumed to be a variant of follicular thyroid carcinoma. It represents only $3 \%$ of all thyroid cancers [4]. It is also known as oxyphilic cell carcinoma. Its prognosis is worse than follicular thyroid carcinoma [5]. Management of hurthle cell carcinoma is same as of follicular carcinoma. Few exceptions are there. Firstly loco-regional nodal involvement is more common, so therapeutic nodal dissection is always needed. Secondly metastatic hurthle cell carcinoma is less likely to concentrate RAl131. Post op RAl131 can be considered in patients with gross residual disease or patients with suspected metastasis. RAI can be considered after thyroidectomy if post op stimulated $\mathrm{Tg}$ levels are more than $10 \mathrm{ng} / \mathrm{ml}$ with negative scan. It has been reported to decrease loco-regional recurrence. $\mathrm{RAI}$ is also recommended for unresectable disease with positive imaging. 
In a study done at Memorial Sloan-Kettering Cancer center [6], outcomes of 56 patients with hürthle cell cancer were analyzed. In this study, recurrence was a significant predictor of tumor-related mortality, and the most significant predictor of outcome was extent of invasion. In addition, tumor size, extrathyroidal disease extension, and initial nodal or distant metastasis were found to be associated with an adverse outcome [7].

Hürthle cell cancer has the highest incidence of metastasis among the differentiated thyroid cancers. Metastatic disease is reported at the time of initial diagnosis in $10-20 \%$ of patients and in $34 \%$ of the patients overall.

\section{REFERENCES}

1. Tai P, Korzeniowski M, Sadikov E, et al. Issues in managing hrthle cell carcinoma of thyroid:a case report. Cureus 2017; 9(4): 1167.
2. Oluic B, Paunovic I, Loncar Z, et al. Survival and prognostic factors for survival, cancer specific survival and disease free interval in 239 patients with Hurthle cell carcinoma: a single center experience. Bmc Cancer 2017; 17(1): 371.

3. Tuttle RM, Haugen B, Perrier ND. Updated American Joint Committee on cancer/tumor-node-metastasis staging system or differentiated and anaplastic thyroid cancer: what changed and why? Thyroid 2017; 27(6): 751-6.

4. Hundahl SA, Fleming ID, Fremgen AM, et al. A national cancer data base report on 53,856 cases of thyroid carcinoma treated in the U.S., 1985-1995. Cancer 1998; 83(12): 2638-48.

5. Goffredo P, Roman, SA, Sosa JA. Hurthle cell carcinoma: a population-level analysis of 3311 patients. Cancer 2013; 119: 50411.

6. Stojadinovic A, Ghossein RA, Hoos A, et al. Hürthle cell carcinoma: a critical histopathologic appraisal. J Clin Oncol 2001; 19(10): 2616-25.

7. Strazisar B, Petric R, Sesek M, et al. Predictive factors of carcinoma in 279 patients with hürthle cell neoplasm of the thyroid gland. J Surg Oncol 2010; 101(7): 582-6. 